\title{
The metabolomic profile of red non- $V$. vinifera genotypes
}

\author{
Silvia Ruocco ${ }^{\mathrm{a}, \mathrm{b}}$, Marco Stefanini ${ }^{\mathrm{c}}$, Jan Stanstrup ${ }^{\mathrm{a}}$, Daniele Perenzoni ${ }^{\mathrm{a}}$, Fulvio Mattivi ${ }^{\mathrm{a}}$, Urska Vrhovsek ${ }^{\mathrm{a}, *}$ \\ a Department of Food Quality and Nutrition, Research and Innovation Centre, Fondazione Edmund Mach, Via E. Mach 1, 38010 San Michele all'Adige, Italy \\ ${ }^{\mathrm{b}}$ Department of Agricultural, Food, Environmental and Animal Sciences, University of Udine, Via delle Scienze 208, 33100 Udine, Italy \\ c Department of Genomics and Biology of Fruit Crop, Research and Innovation Centre, Fondazione Edmund Mach, Via E. Mach 1, 38010, San Michele all'Adige, Italy
}

\section{A R T I C L E I N F O}

\section{Article history:}

Received 25 November 2016

Received in revised form 24 January 2017

Accepted 27 January 2017

Available online 30 January 2017

\section{Keywords:}

Wild grapevine

Grape

Metabolomic fingerprint

Polyphenols

Lipids

\begin{abstract}
A B S T R A C T
Wild American genotypes represent an important part of the Vitis germplasm in relation to grape improvement. Today, these genotypes are currently involved in breeding programmes in order to introgress traits resistant to pests and diseases in $V$. vinifera cultivars. Nevertheless, the metabolic composition of their grapes has not been widely investigated. This study aimed to explore in detail the metabolomic profile in terms of simple phenolic, proanthocyanidin, anthocyanin and lipid compounds in two hybrids and five American genotypes. The results were compared with those of two $V$. vinifera cultivars. A multi-targeted metabolomics approach using a combination of LC-MS and LC-DAD methods was used to identify and quantify 124 selected metabolites. The genotypes studied showed considerable variability in the metabolomic profile according to the grape composition of $V$. vinifera and other Vitis genotypes. As regards the composition of anthocyanins, not all wild genotypes contained both mono- and di-glucoside derivatives. Wild genotype 41B and $V$. vinifera cultivars contained only monoglucoside anthocyanins. The proanthocyanidins of non- $V$. vinifera genotypes were mainly rich in oligomers and short-chain polymers. The analysis of lipids in wild Vitis genotypes, here reported for the first time, showed the existence of a certain diversity in their composition suggesting a strong influence of the environmental conditions on the general lipid pattern.
\end{abstract}

@ 2017 Elsevier Ltd. All rights reserved.

\section{Introduction}

Grape is one of the most widely distributed fruits in the world which is mainly used for the production of wine, juice and raisins. Given the chemical diversity of different grape metabolomes, considerable effort and research should be dedicated to studying the grape composition of wild Vitis genotypes, which represent an important part of the Vitis germplasm. Indeed, the Vitis genus accounts for many uncultivated species, widely distributed in southern Europe, Asia Minor, eastern Asia and North and Central America (Arnold, Schnitzler, Douard, \& Peter, 2007). Among these, wild American genotypes include a large number of species characterized by small berries with abundant seeds and strong pungent flavours such as the foxy flavour (Vivier \& Pretorius, 2000). In particular, foxy compounds have been described in $V$. labrusca and $V$. rotundifolia but not in other American Vitis species (Sun, Gates, Lavin, Acree, \& Sacks, 2011). Furthermore, non-V. vinifera genotypes were also shown to have high resistance to major grapevine diseases such as powdery and downy mildew. This characteristic captured the attention of grape breeders, because it was considered to offer an important solution to the environmental problems caused by intense and systematic use of chemical products to protect $V$. vinifera crops. Indeed, since

\footnotetext{
* Corresponding author.

E-mail address: urska.vrhovsek@fmach.it (U. Vrhovsek).
}

the 19th century wild American Vitis species have been used in breeding programmes in order to combine the resistant traits of American species with the grape quality of $V$. vinifera cultivars, resulting in the development of interspecific hybrids.

Although today wild American genotypes represent an important source of variability in the Vitis genus and have considerable agricultural importance, little research has been conducted on their grape composition. A few studies have investigated the phenolic pattern of non- $V$. vinifera genotypes (Acevedo De la Cruz et al., 2012; Hilbert et al., 2015; Liang, Yang, Cheng, \& Zhong, 2012; Narduzzi, Stanstrup, \& Mattivi, 2015; Zhao, Duan, \& Wang, 2010). Recent advances in metabolomics offer the chance to obtain a comprehensive view of the metabolites present in samples using combined targeted analytical techniques. The objective of this work was to characterise the metabolic composition of seven non- $V$. vinifera grape berries from different vintages in terms of quantity and pattern of polyphenols and lipids. To our knowledge, for the first time, the lipid composition of wild American genotypes has been investigated.

Polyphenols represent one of the largest classes of secondary metabolites in the grape, known to contribute to wine quality and to play a significant role in human nutrition and health (Xia et al., 2010). Anthocyanins, mainly present in the skin, are the pigments responsible for the colour of red grapes. Flavan-3-ols, the basic building blocks of grape tannins, represent the most abundant class of polyphenols in 
Table 1

List of genotypes included in this study.

\begin{tabular}{|c|c|c|c|}
\hline Genotype name & Original pedigree & Species & Vintage \\
\hline 41B & $V$. vinifera Chasselas $\times V$. berlandieri & American hybrid & $2008,2009,2010$ \\
\hline Kober 5BB & $V$. berlandieri $\times V$. riparia & American hybrid & $2007,2008,2009,2010,2014$ \\
\hline V. andersonii & V. andersonii & V. andersonii & $2007,2008,2009,2010$ \\
\hline V. champinii & V. champinii & V. champinii & $2007,2008,2009,2010,2013$ \\
\hline$V$. cinerea & V. cinerea Engelmann & V. cinerea & $2008,2010,2013,2014$ \\
\hline V. californica & V. californica & V. californica & $2007,2008,2010,2013,2014$ \\
\hline V. arizonica Texas & V. arizonica Engelmann & V. arizonica Texas & $2007,2008,2009,2014$ \\
\hline Pinot Noir & & V. vinifera & $2007,2008,2009,2010$ \\
\hline Cabernet Sauvignon & & V. vinifera & 2007, 2008, 2009, 2010 \\
\hline
\end{tabular}

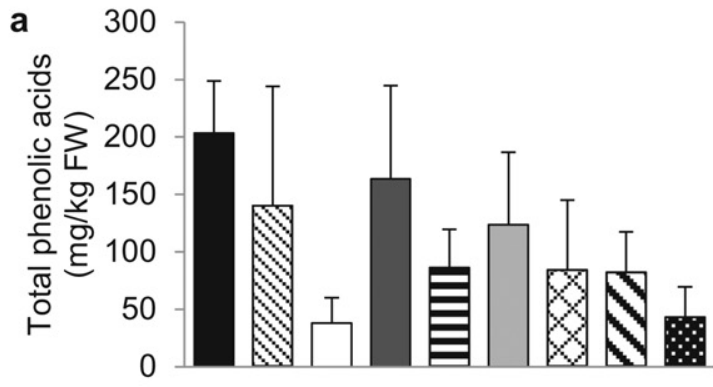

C
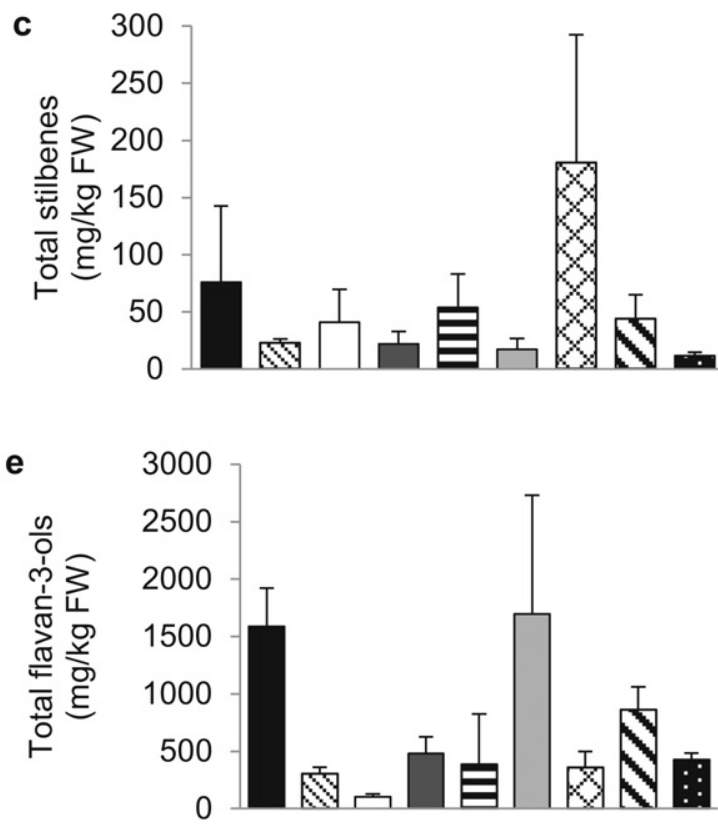
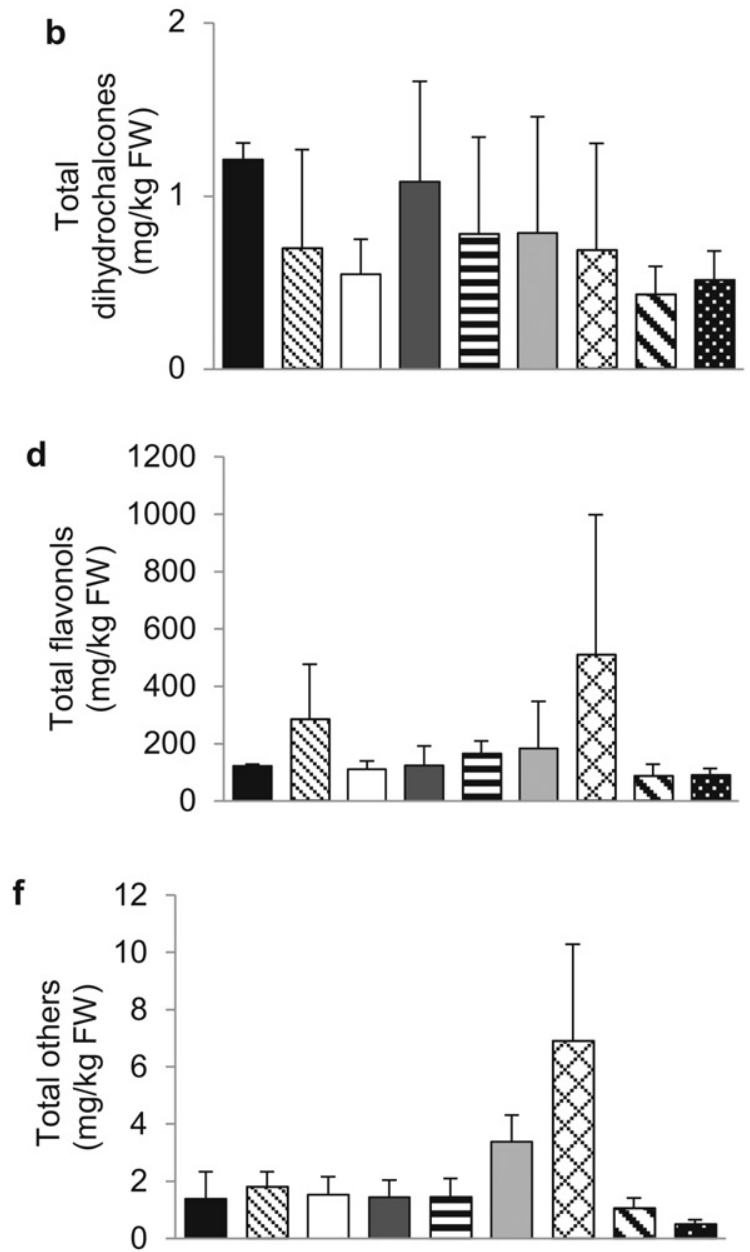

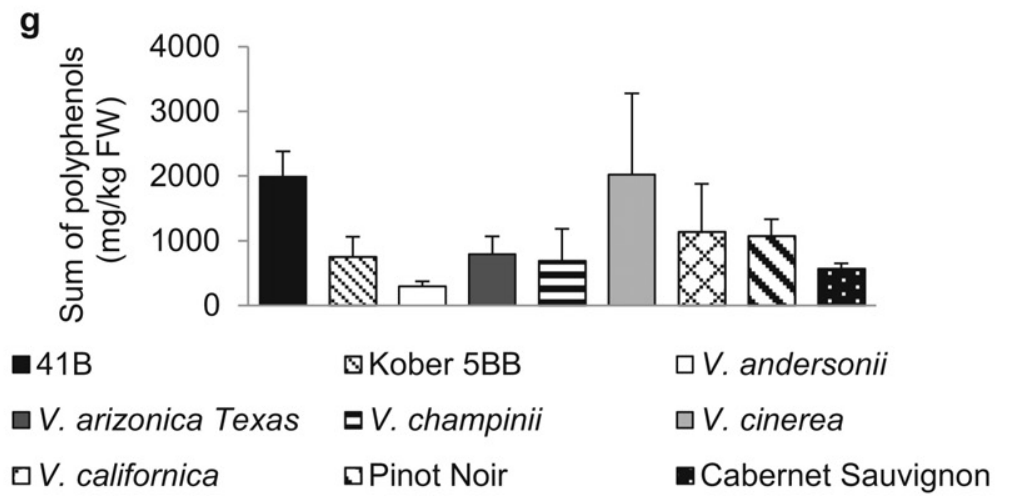

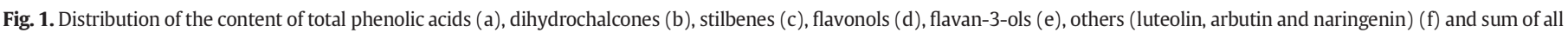
the polyphenols ( $\mathrm{g}$ ). 
grape berries. These metabolites are primarily located in the seeds and skins and their content is very important since they are responsible for the astringency and bitterness of red wines (Teixeira, Eiras-Dias, Castellarin, \& Gerós, 2013). Stilbenes are phytoalexins produced in response to biotic stress, such as the grape pathogens Plasmopara viticola and Botrytis cinerea, or abiotic stress (Pezet, Gindro, Viret, \& Spring, 2004a).

Another important class of metabolites is made up of lipids, which represent a diverse and ubiquitous group of compounds with many key biological functions, including acting as structural components of cell membranes, providing energy for metabolic activities and participating in signalling events (Fahy, Cotter, Sud, \& Subramaniam, 2011). In particular, grape lipids are important factors in oenology since they are capable of modulating the yeast metabolism during the winemaking process (Delfini \& Cervetti, 1991).

\section{Materials and methods}

\subsection{Chemicals}

Methanol (LC-MS and HPLC grade), acetonitrile (LC-MS grade), 2propanol, chloroform and phloroglucinol were purchased from SigmaAldrich (Milan, Italy). Formic acid and ammonium formate additives for LC-MS were from Fluka Sigma-Aldrich (Milan, Italy). Water was of Milli-Q grade obtained from a Millipore system (Millipore, Billerica, MA, USA). Chemical standards were purchased or isolated as reported by the corresponding method used for the analysis of each class of metabolites.

\subsection{Grapes}

In the present work, grape samples of two hybrid varieties, five American Vitis species and two V. vinifera cultivars were studied (Table 1). Grape berries were harvested at technical maturity in the Fondazione Edmund Mach vineyards (San Michele all'Adige, Trento, Italy) in 6 different vintages. The samples were immediately frozen and stored at $-80^{\circ} \mathrm{C}$. Then, grape berries were ground under liquid nitrogen using an analytical mill (IKA, Germany) to obtain a frozen powder.

\subsection{Extraction procedures}

\subsubsection{Phenolic compounds}

The phenolic compounds were extracted according to Vrhovsek et al. (2012). Two grams of grape powder were extracted twice with a solvent mixture of water, methanol and chloroform $(20: 40: 40 \mathrm{v} / \mathrm{v})$. The supernatants from two extractions were combined and filtered into an LC-MS vial.

\subsubsection{Anthocyanins}

The anthocyanins were extracted according to Mattivi, Guzzon, Vrhovsek, Stefanini, and Velasco (2006). The skin of 20 frozen berries were subjected two times to extractions with methanol and analysed by LC-MS instrument (Mattivi et al., 2006).

\subsubsection{Proanthocyanidins}

The samples were prepared by a slightly modified version of the method described in Gris et al., 2011 and Narduzzi et al., 2015. Briefly, $1 \mathrm{~g}$ of grape powder was extracted twice with $4 \mathrm{~mL}$ of methanol for $15 \mathrm{~min}$, dried in a rotavapor and reconstituted with $20 \mathrm{~mL}$ of water. The water extract was loaded onto a C18 Sep-pak, washed with $30 \mathrm{~mL}$ of water, eluted with $15 \mathrm{~mL}$ of methanol, evaporated and then reconstituted with $2 \mathrm{~mL}$ of methanol. One hundred microlitres of the elute was added to $900 \mu \mathrm{L}$ of methanol and water $(50 / 50 \mathrm{v} /$ $\mathrm{v}$ ), filtered, and injected into the LC-MS system. A further one hundred microlitres of concentrated sample were added to $100 \mu \mathrm{L}$ of phloroglucinol reagent at $50{ }^{\circ} \mathrm{C}$ for $30 \mathrm{~min}$ and then combined with $1 \mathrm{~mL}$ of sodium acetate to stop the reaction. The samples were filtered and immediately analysed.

\subsubsection{Lipids}

The samples were prepared according to Della Corte et al. (2015) by extraction with chloroform. Each sample was injected two times, non diluted and diluted 1:100 with acetonitrile, isopropanol and water $(65: 30: 5 \mathrm{v} / \mathrm{v} / \mathrm{v})$, in order to allow quantification of the most abundant lipids (Della Corte et al., 2015).
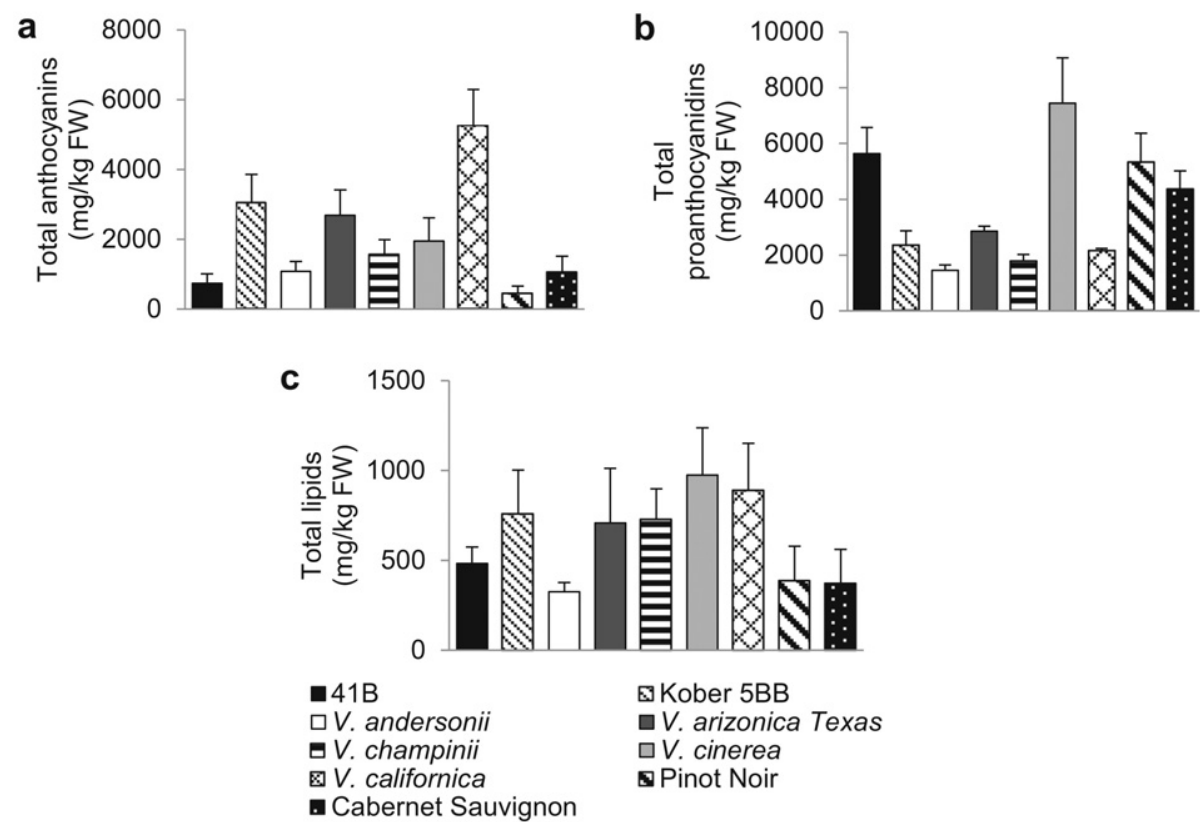

Fig. 2. Distribution of the content of total anthocyanins (a), proanthocyanidins (b) and lipids (c). 


\subsection{Analysis of compounds}

\subsubsection{Analysis of phenolic compounds}

Analysis of phenolic compounds was performed as previously described (Vrhovsek et al., 2012). A Waters Acquity UPLC system ${ }^{\circledR}$ (Milford, MA, USA) was used. Phenolic compounds were separated on a Waters Acquity HSS T3 column $(1.8 \mu \mathrm{m}, 100 \times 2.1 \mathrm{~mm}$; Milford, MA, USA), thermostated at $40{ }^{\circ} \mathrm{C}$. The mobile phase was composed of component $A$ ( $0.1 \%$ formic acid in water) and component $B(0.1 \%$ formic acid in acetonitrile).

Mass spectrometry detection was performed on a Waters Xevo TQMS ${ }^{\circledR}$ (Milford, MA, USA) instrument equipped with an electrospray (ESI) source. Further MS parameters are reported in Vrhovsek et al. (2012). Polyphenol concentrations were calculated in milligrams per kilogram ( $\mathrm{mg} / \mathrm{kg}$ ) of fresh weight (FW) by means of calibration curves and using gentisic and rosmarinic acids as internal standards.

\subsubsection{Analysis of anthocyanins}

HPLC separation and quantification of anthocyanins was carried out on a Waters 2695 separation module equipped with Waters 996 DAD detector (Milford, MA, USA). Anthocyanins were quantified at $520 \mathrm{~nm}$ using a calibration curve with malvidin 3-glucoside and expressed as $\mathrm{mg} / \mathrm{kg}$ FW (Mattivi et al., 2006).

\subsubsection{Analysis of proanthocyanidins}

Analysis of condensed tannins was performed with Waters Acquity UPLC $®$ system, coupled with Waters Xevo TQMS® (Milford, MA, USA). Chromatographic, separation and detection conditions were the same as reported above (Vrhovsek et al., 2012). Catechin, epicatechin, procyanidins B1 and B2, gallocatechin, epigallocatechin and epicatechin gallate were quantified using a linear regression curve built on the injection of pure chemical standards. Quantification of phloroglucinol-bound flavanols was done as for epicatechin, epigallocatechin, and epicatechin gallate equivalents, respectively. The results were expressed as $\mathrm{mg} / \mathrm{kg}$ FW (Gris et al., 2011).

\subsubsection{Analyisis of lipids}

Analysis was carried out on a UHPLC Dionex 3000 (Thermo Fisher Scientific, Germany) connected to an API 5500 triple-quadrupole mass spectrometer (Applied Biosystems/MDS Sciex, Toronto, Canada) equipped with an electrospray source. For the separation of compounds a reversed phase column Ascentis Express C18 $(15 \mathrm{~cm} \times 2.1 \mathrm{~mm}$, $2.7 \mu \mathrm{m}$; Sigma, Italy) was used. The solvents were: solvent A

\section{b}
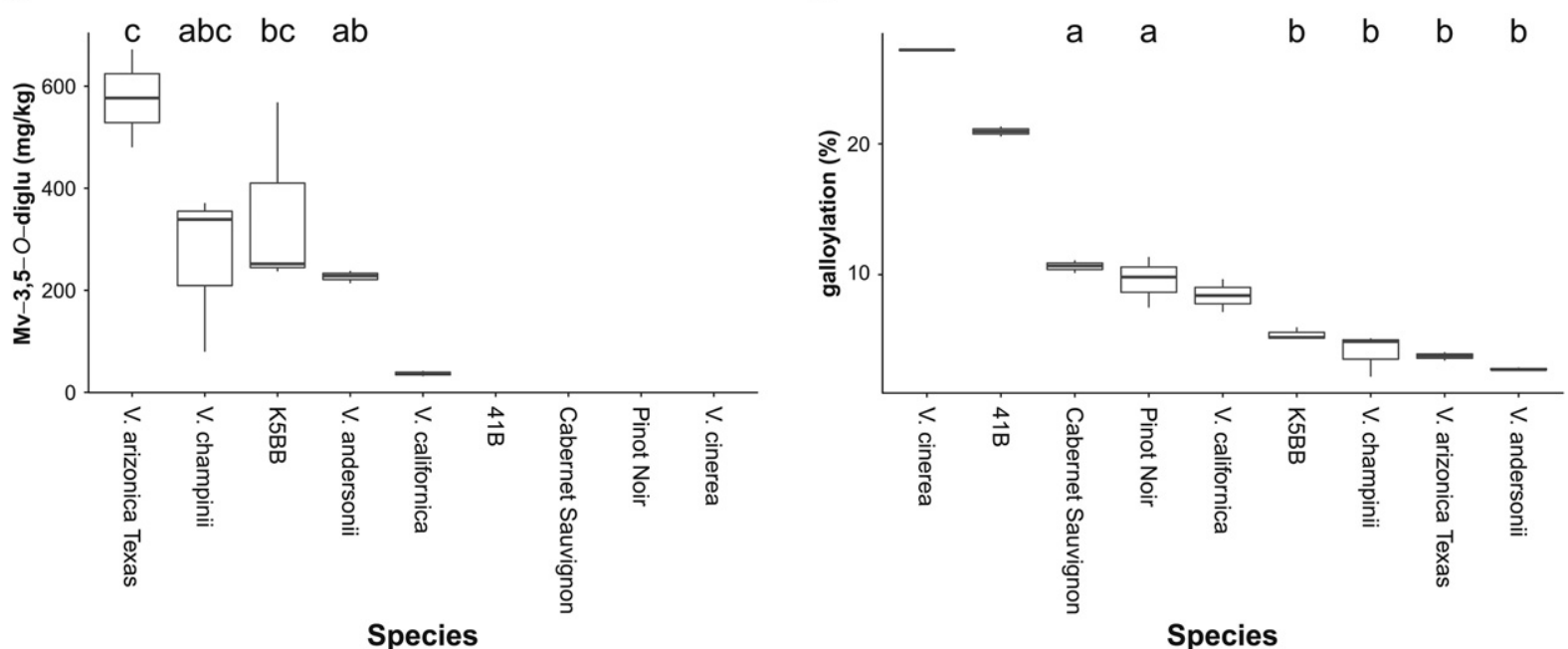

C

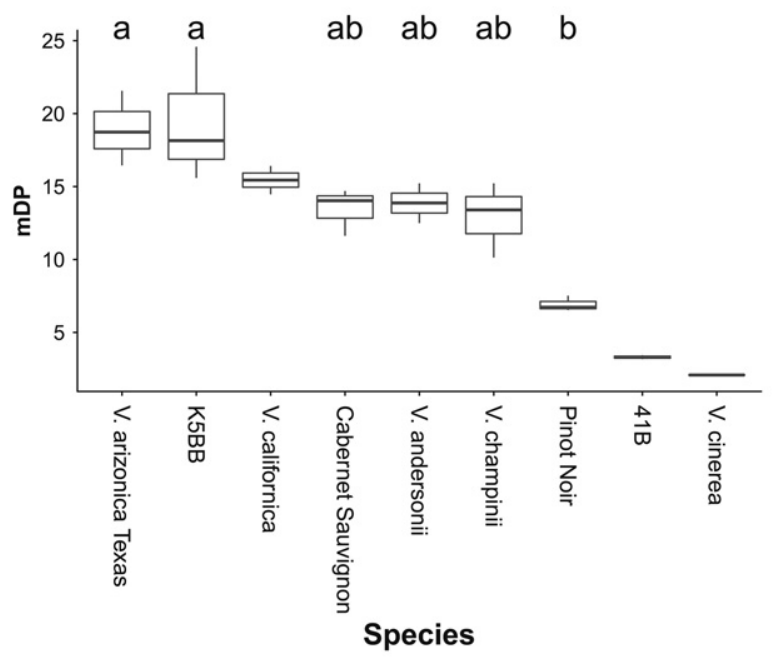

d

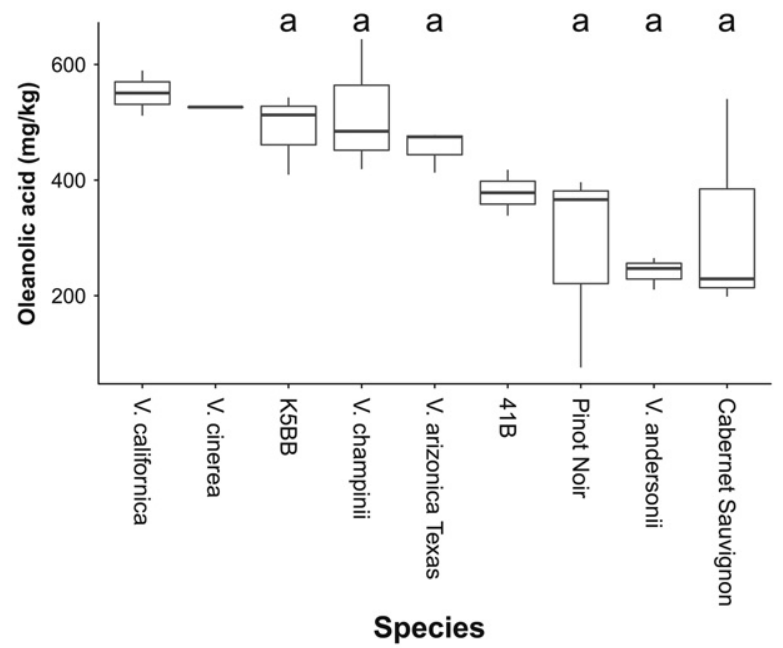

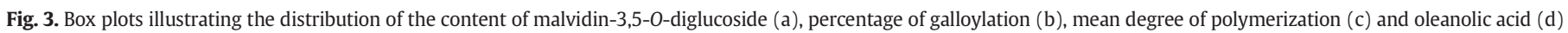
measured for the vintages 2007, 2008 and 2009; different letters indicate significant different groups in analysis of variance (ANOVA). 
(acetonitrile $40 \%$ in water, ammonium formate $10 \mathrm{mM}$ and formic acid $0.1 \%$ ) and solvent B (isopropanol $90 \%$, acetonitrile $10 \%$, ammonium formate $10 \mathrm{mM}$ and formic acid $0.1 \%$ ).

The target lipids were detected under multiple reaction monitoring (MRM) mode and identified on the basis of their reference standard, retention time and qualifier and quantifier ion. Quantification was carried out using calibration curves for each analyte and data were expressed as $\mathrm{mg} / \mathrm{kg} \mathrm{FW}$ after normalization on the basis of the internal standard docosahexaenoic acid (Della Corte et al., 2015).

\subsection{Statistical analysis}

All data analysis was performed using R v3.3.1 (R Development Core Team, 2014). The quantitative data recorded was analysed using a fixed effects linear model with species as the fixed effect. The years were thus considered replicates. Prior to modelling zero intensity values (below the limit of quantification) were replaced compound-wise by a random number between the lowest detected intensity and zero. A model was fitted for each compound and these models used for pairwise comparisons between all species without correction for multiple testing. Subsequently, the collection of p-values for all comparisons were corrected for multiple testing by controlling the local false discovery rate (FDR) and q-values calculated (Strimmer, 2008a, 2008b). Unit variance scaling was used for each compound for both PCAs and heatmaps. For heatmaps the Pearson correlation coefficient and Ward's minimum variance methods were used for hierarchical clustering (Murtagh \& Legendre, 2014). Missing values were replaced as above for the dendrogram calculations and marked in grey in these figures. Values outside the range of 3 standard deviations were reassigned to 3. For PCA, compounds with more than $50 \%$ missing values were excluded and PCA was performed using the NIPALS method (Stacklies, Redestig, Scholz,
Walther, \& Selbig, 2007; Wold, 1966). The sample of V. champinii in 2007 was a clear outlier in the lipid analysis. The sample has therefore been removed in the reported PCA plots. The complete data analysis is available at: https://github.com/stanstrup/vinifera_ruocco.

\section{Results}

\subsection{Phenolic profiles}

In total, 57 phenolic compounds were analysed (Table S1) and the corresponding box plots are shown in the Supplementary material 1. The total content of the main chemical classes of phenolic compounds (phenolic acids, dihydrochalcones, stilbenes, flavonols, flavan-3-ols and others) determined in grape berries is shown in Fig. 1. In wild genotypes the phenolic acid content ranged from 20 to $265 \mathrm{mg} / \mathrm{kg}$ FW (Fig. 1a). In contrast, phenolic acids varied from 18 to $133 \mathrm{mg} / \mathrm{kg}$ FW in V. vinifera cultivars. The dihydrochalcones phloridzin and phloretin were found in small amounts in all genotypes (Fig. 1b). V. californica was the genotype with the highest mean level of total stilbenes (180 mg/kg FW) compared to all other genotypes (Fig. 1c). The total flavonol content ranged from 14 to $1032 \mathrm{mg} / \mathrm{kg} \mathrm{FW}$, showing considerable variation in different genotypes and vintages (Fig. 1d). As regards the total content of flavan-3-ols, it ranged from 335 to $1148 \mathrm{mg} / \mathrm{kg} \mathrm{FW}$ in $V$. vinifera cultivars, and from 61 to $3120 \mathrm{mg} / \mathrm{kg}$ FW in wild genotypes (Fig. 1e). Other phenolic compounds, such as luteolin, arbutin and naringenin, were found in small amounts (Fig. 1f). The sum of all analysed phenols in $V$. vinifera cultivars ranged from 445 to $1426 \mathrm{mg} / \mathrm{kg} \mathrm{FW}$, while it varied from 182 to $3695 \mathrm{mg} / \mathrm{kg}$ FW in wild genotypes, with differences between genotypes and vintages. $V$. cinerea and $V$. andersonii had the highest and lowest average values over

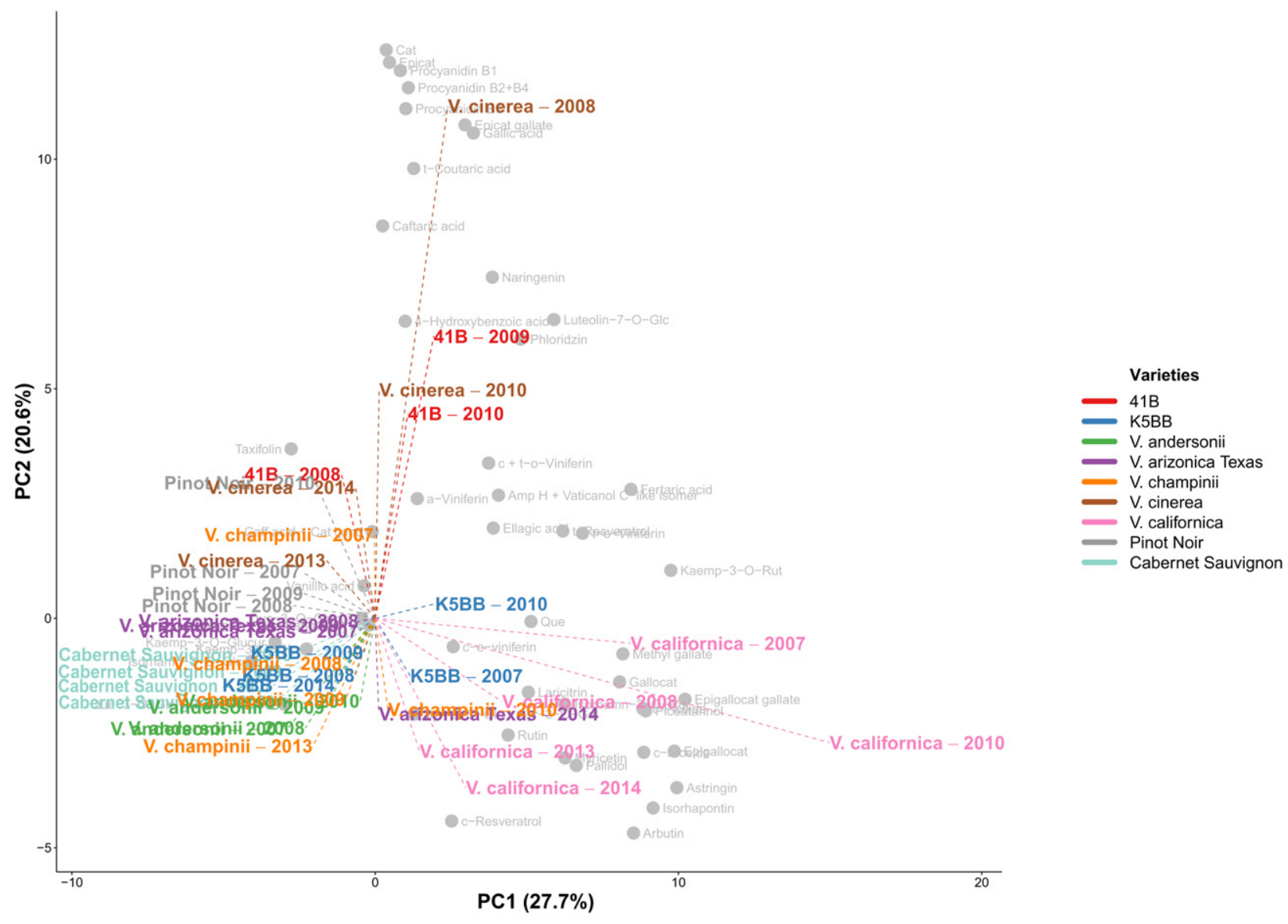

Fig. 4. PCA plot of simple phenols with distribution of grape samples. See Table S1 for the list of abbreviations of the compounds identified. 
different vintages for total phenols (2025 and $296 \mathrm{mg} / \mathrm{kg}$ FW respectively) (Fig. 1g).

\subsection{Anthocyanin profiles}

Total anthocyanins varied significantly with the genotype (Fig. 2a). $V$. californica and 41B had the highest and lowest mean anthocyanin content within wild genotypes (5244 and $735 \mathrm{mg} / \mathrm{kg}$ FW respectively). In contrast, $V$. vinifera cultivars such as Cabernet Sauvignon and Pinot Noir had a lower mean amount of anthocyanins (1045 and $437 \mathrm{mg} / \mathrm{kg}$ FW respectively). The results and the box plots of the 20 anthocyanins detected in the samples are shown in Table S1 and Supplementary material 1, respectively. Malvidin derivatives were the most abundant components of total anthocyanins in Cabernet Sauvignon, Pinot Noir, $V$. cinerea and 41B. Conversely, delphinidin derivatives were the most abundant components in Kober 5BB, V. andersonii, V. californica, V. arizonica Texas and V. champinii. In V. vinifera cultivars all the anthocyanins detected were monoglucoside derivatives. In contrast, in wild Vitis genotypes diglucoside anthocyanins were found in different amounts: 41B did not contain diglucosides, $V$. cinerea and $V$. californica contained less than $5 \%$ of the total amount of anthocyanins (from 5 to $136 \mathrm{mg} / \mathrm{kg}$ FW) while Kober 5BB, V. andersonii, V. arizonica Texas and $V$. champinii contained more than $40 \%$ of the total (from 522 to $2658 \mathrm{mg} / \mathrm{kg}$ FW). In Fig. 3a the distribution of the malvidin 3,5diglucoside content determined in the samples is presented as a box plot.

Non-acylated anthocyanins were the most abundant compounds in all the samples. In wild genotypes they ranged from about $93 \%$ in $V$. californica to $98 \%$ in $V$. arizonica Texas; while in $V$. vinifera cultivars non-acylated anthocyanins accounted for about $80 \%$ in Cabernet Sauvignon and 100\% in Pinot Noir. Acetyl derivatives were the most abundant compounds in Cabernet Sauvignon (16\%) and Kober 5BB (2\%) as compared to coumaroyl derivatives in all the other genotypes. In Pinot Noir neither acetyl nor coumaroyl derivatives were detected (Table S1).

\subsection{Proanthocyanidin profiles}

The highest mean amount of proanthocyanidins was found in $V$. cinerea $(7446 \mathrm{mg} / \mathrm{kg} \mathrm{FW})$ for wild genotypes and in Pinot Noir ( $5300 \mathrm{mg} / \mathrm{kg} \mathrm{FW}$ ) for $V$. vinifera cultivars (Fig. 2b). The proanthocyanidin composition of the genotypes analysed is presented in Table S1. Catechin was the most abundant flavan-3-ol terminal unit in Pinot Noir, Cabernet Sauvignon, 41B, Kober 5BB 2014, V. arizonica Texas 2007 and 2008, V. cinerea and V. californica 2008 and 2010. Epigallocatechin was the second most abundant flavan-3-ol terminal unit in all the other wild genotypes with the exception of $V$. californica 2013 and 2014 which contained a high percentage of epicatechins as terminal units. The highest proportion of epicatechin and gallocatechin terminal units were found in V. cinerea and Cabernet Sauvignon, with 30\% and 6\% respectively. Epicatechin gallate was the only gallate-derivative found as an upper unit in all samples.

The flavan-3-ol extension units mainly comprised catechin and epigallocatechin, with the predominance of catechin in $V$. vinifera cultivars and in 41B, Kober 5BB 2007 and 2014, V. arizonica Texas, V. andersonii 2007, V. champinii 2007 and 2008, V. cinerea, V. californica 2008. On the other hand, only in the 2010, 2013 and 2014 vintages of $V$. californica, epicatechin was found to be the most abundant flavan-3-ol extension unit. Gallocatechin was the extension unit present in the lowest concentration in all samples. The percentage of galloylation (\%G) revealed that $V$. cinerea and 41B had a larger percentage of galloylated forms, which accounted for $23 \%$ and $22 \%$ respectively, in comparison

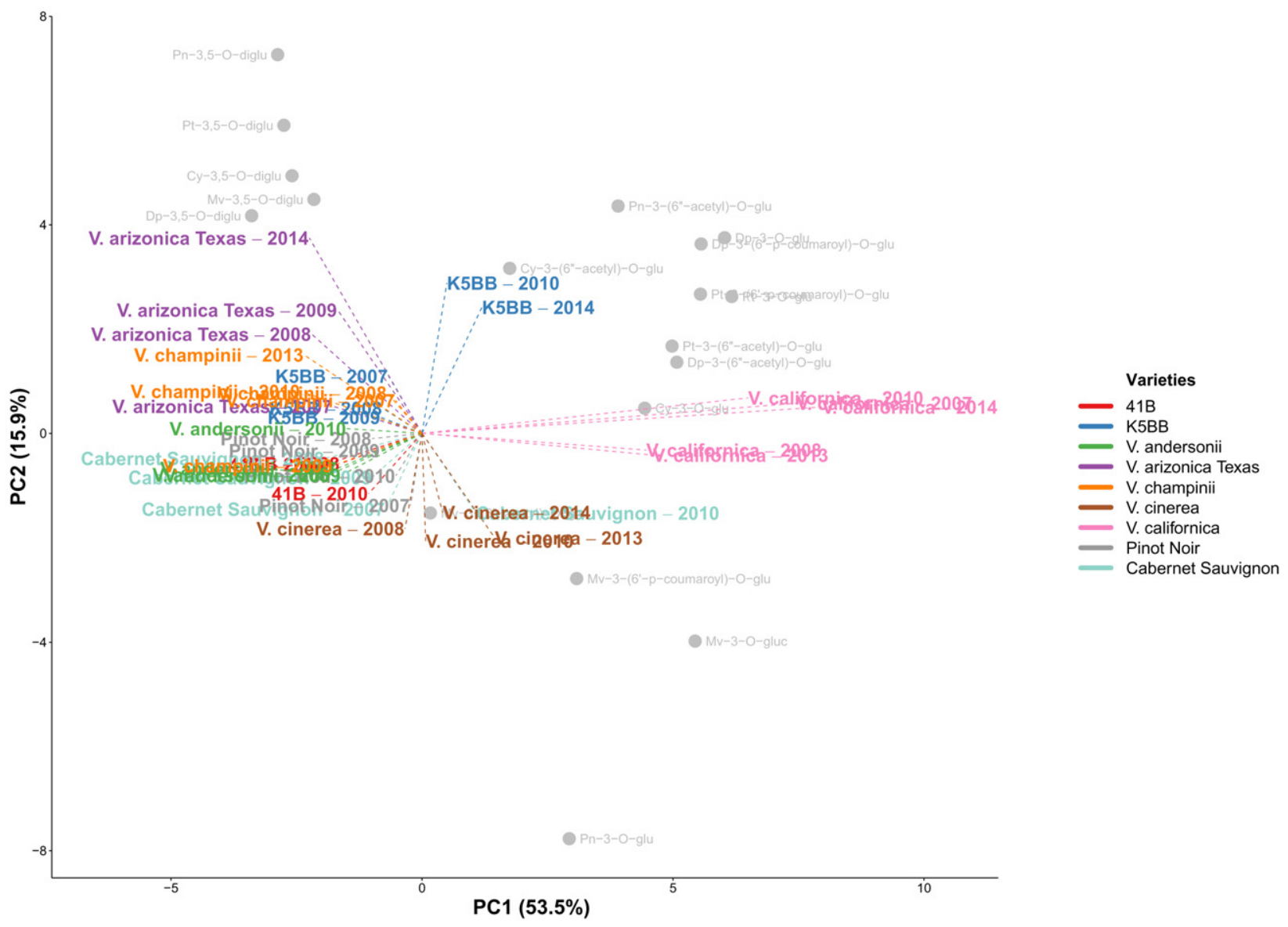

Fig. 5. PCA plot of anthocyanins with distribution of grape samples. See Table S1 for the list of abbreviations of the compounds identified. 
to all other samples (Fig. 3b). The values observed for the mean degree of polymerization ( $\mathrm{mDP}$ ) in the genotypes studied ranged from 2 to 26 units (Fig. 3c). V. cinerea and 41B had the lowest mean degree of mDP polymerization, while $V$. arizonica Texas and Kober 5BB had the highest as compared to all other genotypes. Of the $V$. vinifera cultivars, Cabernet Sauvignon had the highest values of mDP, which ranged from 12 to 16, similar to the values found for $V$. andersonii, V. californica and $V$. champinii. In Supplementary material 1 the box plots of the proanthocyanidins are reported.

\subsection{Lipid profiles}

In this work different classes of grape lipids were studied: fatty acids, sterols, glycerolipids, glycerophospholipids and sphingolipids (Table S1). The total lipid content of wild genotypes ranged from 330 to $1294 \mathrm{mg} / \mathrm{kg} \mathrm{FW}$, while it varied from 114 to $564 \mathrm{mg} / \mathrm{kg} \mathrm{FW}$ in $V$. vinifera cultivars (Fig. 2c). V. cinerea and $V$. andersonii had the highest and lowest mean content of total lipids, 974 and $326 \mathrm{mg} / \mathrm{kg}$ FW respectively. Oleanolic acid was the most abundant compound found in all genotypes, and the distribution can be observed in the box plot (Fig. 3d). V. cinerea and $V$. californica contained the highest mean content of oleanolic acid (734 and $718 \mathrm{mg} / \mathrm{kg}$ FW respectively) while the lowest mean value was found in $V$. andersonii ( $237 \mathrm{mg} / \mathrm{kg} F W$ ). The main fatty acids were palmitic, linolenic, linoleic and stearic acids. In wild genotypes, palmitic acid ranged from 1 to $56 \mathrm{mg} / \mathrm{kg} \mathrm{FW}$, linolenic acid from 0.2 to $41 \mathrm{mg} / \mathrm{kg} \mathrm{FW}$, linoleic from 0.7 to $26 \mathrm{mg} / \mathrm{kg}$ FW and stearic acid from 3 to $23 \mathrm{mg} / \mathrm{kg} \mathrm{FW}$. In contrast, in $V$. vinifera genotypes these fatty acids were found in smaller amounts. Wild genotypes from the 2013 and 2014 vintages showed a larger amount of some lipids, such as 1,2dilinoleoyl-sn-glycero-3-phosphocholine, 1,2-dioleoyl-sn-glycero-3phospho-rac-(1-glycerol) salt, uvaol, 1-linoleoyl-rac-glycerol, glyceril tripalmitoleate, ergosterol, behenic acid, linolenic acid, stearic acid, lignoceric acid, erucic acid, arachidic acid, oleanolic acid, 1,2,3tripentadecanoylglycerol, and cis-11-eicosenoic acid. The box plots of the lipids analysed are shown in Supplementary material 1.

\subsection{Multivariate analysis}

The data obtained were analysed using multivariate methods in order to evaluate similarities and differences in the metabolomic composition in the genotypes studied. The PCA scores plot of the samples shows that there was good grouping of the genotypes for the different vintages based on the phenolic profile (Fig. 4). This can also be seen in Fig. S1, where cluster analysis shows the existence of year-to-year variations for some genotypes, such as $V$. cinerea, $V$. champinii, 41B and $V$. californica.

In Fig. 5 the first PC distinguished anthocyanin monoglucosides and the rest of the anthocyanins; indeed the grapes of $V$. arizonica Texas, $V$. champinii, $V$. andersonii and Kober 5BB were located in the left part of PC2, explained by their high diglucoside derivative content. Cluster analysis showed that the anthocyanin profiles for each genotype were relatively stable (Fig. S2).

Examination of the PCA scores plot (Fig. 6) shows that V. cinerea, 41B and Pinot Noir samples grouped together in the most right-hand part of the score plot, as a consequence of their higher levels of proanthocyanidins (with the sole exception of epigallocatechin, situated slightly to the left) while the remaining wild genotypes grouped together in the left-hand part of the scatter plot, due to the low proanthocyanidin content. These results can also be seen in the heatmap (Fig. S3), since clustering analysis grouped V. cinerea, 41B, Pinot Noir and Cabernet Sauvignon together, indicating that the proanthocyanidin patterns were similar.

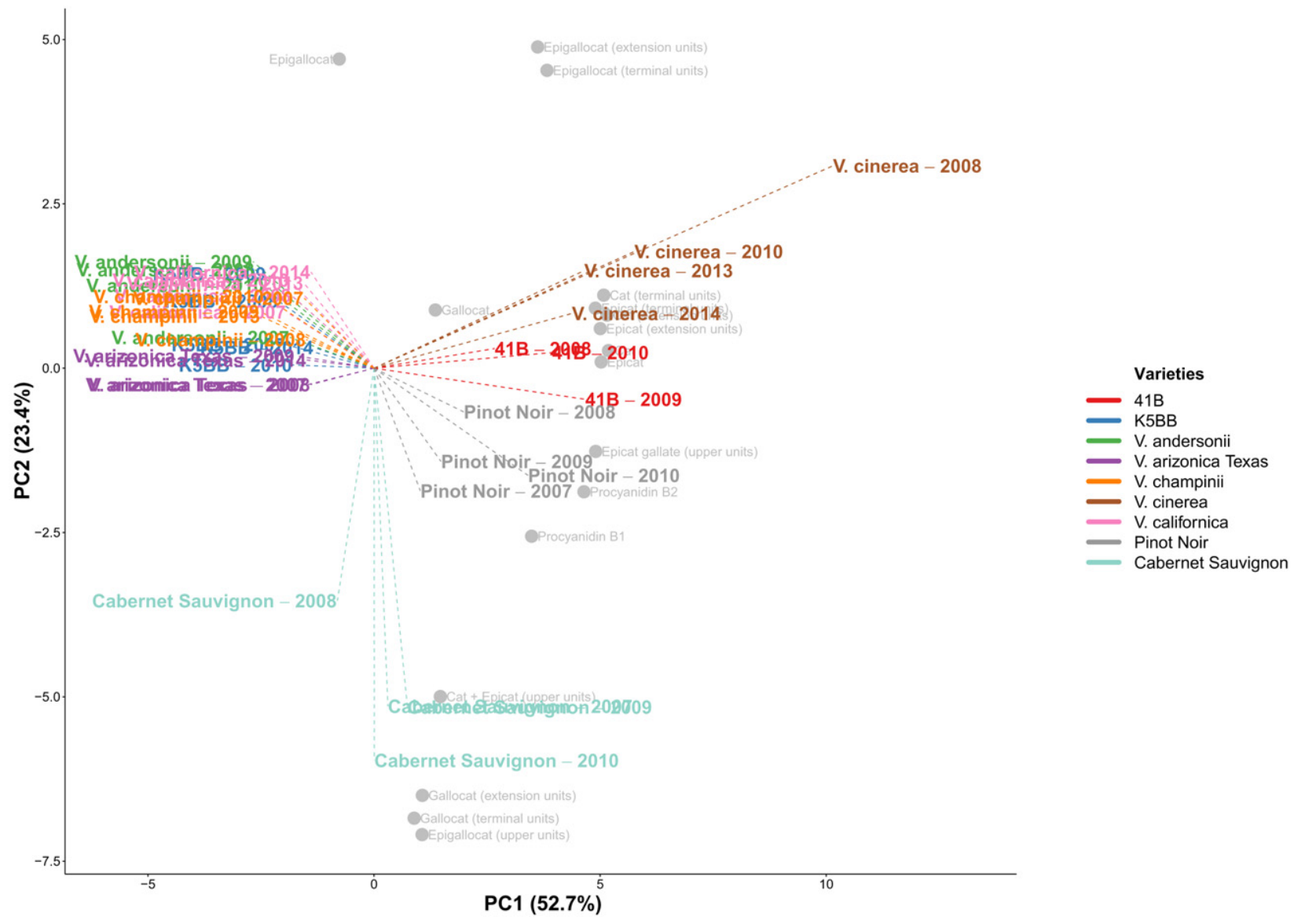

Fig. 6. PCA plot of proanthocyanidins with distribution of grape samples. See Table S1 for the list of abbreviations of the compounds identified. 
The PCA analysis results of lipids reveal that most of genotypes did not group together over the years (Fig. 7). In particular, hierarchical clustering showed that only Pinot Noir and Cabernet Sauvignon grouped together over the years (Fig. S4). Furthermore, it can be observed that wild genotypes of the 2013 and 2014 vintages were closely grouped together.

\section{Discussion}

The grape metabolome is very complex and involves several different classes of compounds. Knowledge about the grape metabolites of non- $V$. vinifera genotypes is still incomplete, since these genotypes have received less attention than $V$. vinifera cultivars. In this context, the present work contributes to bridging this gap in knowledge by analysing the phenolic, anthocyanin, tannin and lipidic profiles of seven non- $V$. vinifera genotypes and two $V$. vinifera cultivars using a targeted metabolomics approach.

The novelty of this study lies in its exhaustive and accurate investigation of a multitude of compounds, in total 124, from different chemical classes. The genotypes included in this study can be directly compared because the impact of soil and climate in the same year was not influential since all genotypes were grown in the same experimental vineyard. Furthermore, the comparison of different years allows to evaluate the influence of climatic conditions among different vintages on the metabolic composition of grape.

\subsection{Phenolic composition}

In this study, the polyphenolic content varied significantly in the genotypes in agreement with Liang et al. (2012) (Figs. 1,2). The accumulation of phenolic compounds in grape berries is significantly affected by genetic and also by non-genetic factors such as environmental conditions (Teixeira et al., 2013), pathogen invasion (Zhu, Zhang, \& Lu, 2012) and generally in response to biotic and abiotic stresses (Jeandet et al., 2010). Resveratrol, an important phytochemical with many well-known benefits for human health, was not found in all the vintages of the genotypes considered, with the exception of Pinot Noir. Pezet, Gindro, Viret, and Richter (2004b) reported that resistant genotypes transform resveratrol into viniferins which are involved in the resistance mechanism of the grapevine to Plasmopara viticola. Later, Malacarne et al. (2011), have observed the existence of a strong negative correlation between the concentrations of stilbenoid viniferins in the leaves and the progress of infection. In our study, the mean amount of viniferins was higher in wild genotypes than in $V$. vinifera cultivars, with the exception of $V$. cinerea. In particular, that found in $V$. cinerea was comparable to that of Pinot Noir suggesting that a different resistance mechanism, which does not include the presence of viniferins, could be involved.

The mean total flavonol content of wild genotypes from the different vintages was higher than in $V$. vinifera cultivars, in accordance with other authors (Liang, Owens, Zhong, \& Cheng, 2011; Liang et al., 2012). Quercetin derivatives were the main flavonols found in $V$. vinifera cultivars as previously described (Hilbert et al., 2015; Liang et al., 2012; Mattivi et al., 2006). The main flavonols found in non- $V$. vinifera genotypes were kaempferol, isorhamnetin and quercetin derivatives in contrast to Hilbert et al. (2015) and Liang et al. (2012) who observed that myricetin and quercetin derivatives were the main flavonols. Caftaric acid was the most abundant of all the hydroxycinnamic derivatives in both $V$. vinifera cultivars and wild genotypes as reported by Liang et al. (2011, 2012). Furthermore, they also observed that in the hydroxybenzoic acid group, vanillic acid content was higher than that of gallic acid.

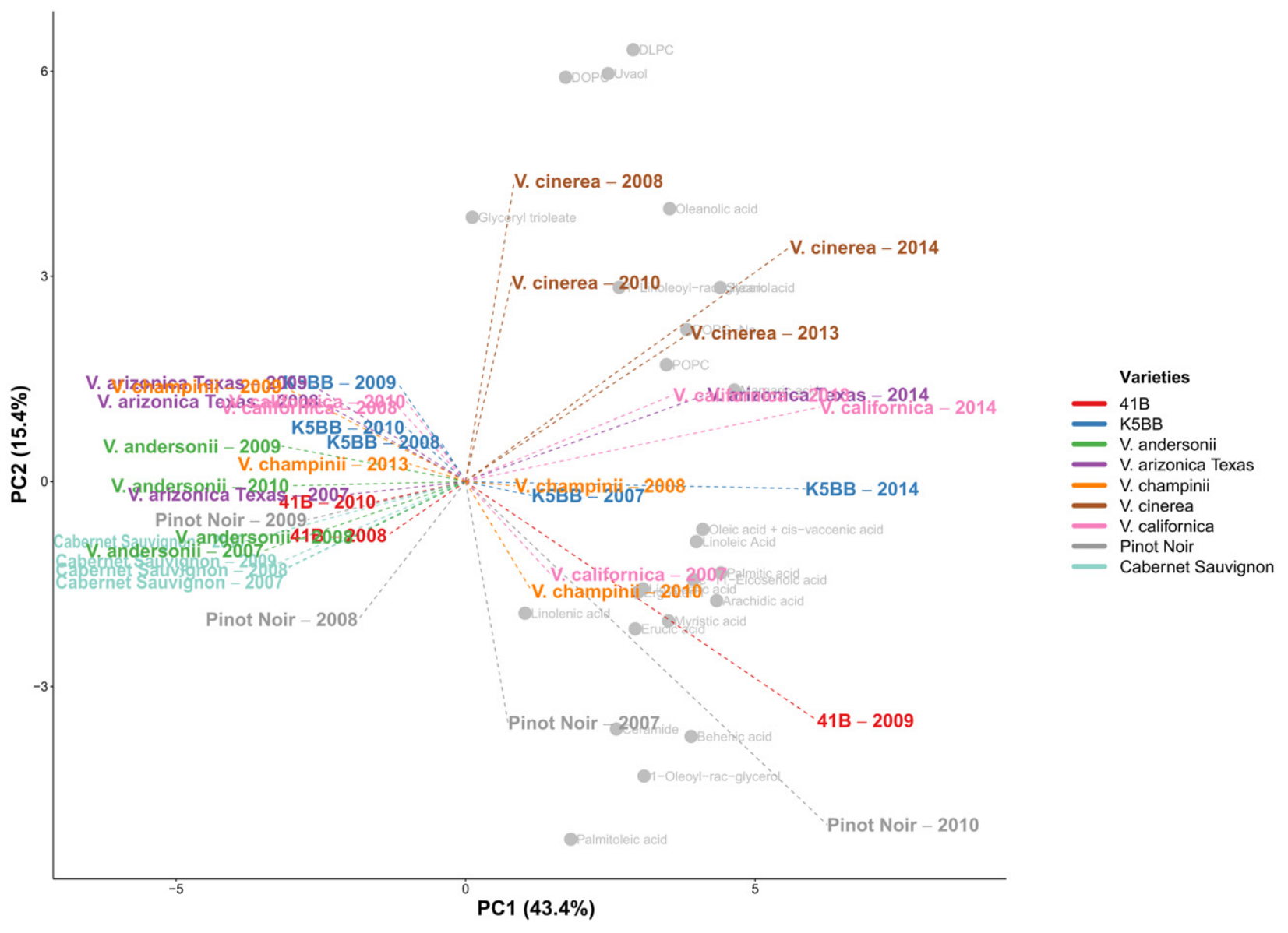

Fig. 7. PCA plot of lipids with distribution of grape samples. See Table S1 for the list of abbreviations of the compounds identified. 
Conversely, we found that gallic acid content was highest in all the samples studied.

Of the dihydrochacones, phloridzin was found in all genotypes, even if it has been demonstrated to be a typical component of apples (Vrhovsek, Rigo, Tonon, \& Mattivi, 2004). Naringenin, known to be one of the main compounds in oranges, mandarins and grapefruits, was also found in small amounts in the genotypes studied (Erlund, 2004). Differences in the relative amount of variation between years were observed for almost all classes of polyphenols, particularly phenolic acids, flavonols and flavan-3-ols and were more significant in wild genotypes than in $V$. vinifera cultivars in agreement with literature (Liang et al., 2012).

\subsection{Anthocyanin composition}

Anthocyanin analysis of the skins showed that the mean content of anthocyanins was higher in non- $V$. vinifera genotypes as compared to $V$. vinifera cultivars, with the exception of the genotype 41B, in agreement with previous studies (Liang et al., 2011, 2012). The relative abundance of anthocyanin derivatives varied from one genotype to another as previously reported (Liang et al., 2012). Furthermore, if the anthocyanin profiles for each genotype were relatively stable, the absolute amounts varied in different vintages due to environmental factors. Diglucosides were not found in 41B while, as expected, the other genotypes had both monoglucoside and diglucoside derivatives since it is known that in wild Vitis species glycosylation occurs at both the 3and 5-positions (Acevedo De la Cruz et al., 2012; Mazzuca, Ferranti, Picariello, Chianese, \& Addeo, 2005). Acevedo De la Cruz et al. (2012) reported that in $V$. cinerea all anthocyanins were monoglucoside derivatives and the anthocyanin levels were similar to those in $V$. vinifera. In our study, the diglucoside content in $V$. cinerea accounted for $1 \%$ of total anthocyanins, while the total anthocyanin amount was higher than in $V$. vinifera cultivars.

\subsection{The influence of proanthocyanidins}

In terms of proanthocyanidins, differences in composition were found in the genotypes analysed. Previously, Narduzzi et al. (2015) and Springer, Sherwood, and Sacks (2016) reported that the total proanthocyanidin content in the skin and seeds of wild American genotypes analysed was lower than in $V$. vinifera cultivars. In our study, $V$. cinerea and 41B accumulated a higher mean content of total proanthocyanidins as compared to both $V$. vinifera cultivars and wild genotypes.

Del Rio and Kennedy (2006) and Mattivi, Vrhovsek, Masuero, and Trainotti (2009) reported that catechin was the most abundant flavan-3-ol terminal unit in Pinot Noir, however in our study it was also found in wild genotypes. Narduzzi et al. (2015) observed a higher mean degree of polymerization in the skin and seeds of $V$. vinifera than in wild American grapes. Anyway the direct comparison between the two studies cannot be done, since in the study of Narduzzi et al. (2015) different $V$. vinifera varieties were analysed and furthermore the grape flesh was not included in the study. According to our results, the proanthocyanidins which have been extracted from whole berries of non- $V$. vinifera genotypes were rich in oligomers and short-chain polymers, with the exception of $V$. cinerea and $41 \mathrm{~B}$. Regarding the percentage of galloylation (\%G), as $41 \mathrm{~B}$ and $V$. cinerea have a lower mDP they showed a proportionally higher abundance of galloylated derivatives in comparison to all other samples. It is known that the bitterness and astringency of wines are affected by the extent of galloylation (Lesschaeve \& Noble, 2005). This work demonstrated that the accumulation of proanthocyanidins varied in wild genotypes and was not significantly influenced by environmental factors.

\subsection{The diversity of lipid profile}

To the best of our knowledge, it is the first time that the class of lipids was analysed in wild American genotypes. Our study showed that non$V$. vinifera genotypes, with the exception of $V$. andersonii, had a higher total lipid content as compared to $V$. vinifera cultivars. In particular, high variability in lipid content of wild genotypes observed in different vintages suggests the strong influence of environmental factors. Stearic, palmitic and linoleic acids were the main fatty acids detected in all genotypes, in general agreement with previous studies (Bauman, Gallander, \& Peng, 1977; Gallander \& Peng, 1980; Tumanov et al., 2015). The presence of oleanolic acid, known to act as "survival factor" for yeasts (Lafon-Lafourcade, Larue, \& Ribéreau-Gayon, 1979), was noted in all genotypes.

\section{Conclusions}

In conclusion, this work provides a comprehensive and systematic survey of the content of all the main classes of metabolites in non- $V$. vinifera genotypes. The results obtained provide clear evidence of (i) differences in anthocyanin composition, since not all wild genotypes are characterized by the presence of diglucosides, (ii) genotypic influence on proanthocyanidin profiles, (iii) the existence of a certain diversity in lipid composition according to the genotype and also with reference to the vintage. To our knowledge, this study is the first detailed and extended survey of non- $V$. vinifera grape metabolites. The information gained could potentially be useful, providing important information for future grapevine breeding.

Supplementary data to this article can be found online at http://dx. doi.org/10.1016/j.foodres.2017.01.024.

\section{Acknowledgements}

This work was financially supported by the ADP 2015 project and PhD scholarship (S.R.), funded by the Autonomous Province of Trento (2016). We thank Domenico Masuero and Evelyn Soini for their assistance in data analysis.

\section{References}

Acevedo De la Cruz, A., Hilbert, G., Rivière, C., Mengin, V., Ollat, N., Bordenave, L., .. Richard, T. (2012). Anthocyanin identification and composition of wild Vitis spp. accessions by using LC-MS and LC-NMR. Analytica Chimica Acta, 732, 145-152.

Arnold, C., Schnitzler, A., Douard, A., \& Peter, R. (2007). Is there a future for wild grapevine (Vitis vinifera subsp. silvestris) in the Rhine Valley? Biodiversity and Conservation, 14, 1507-1523.

Bauman, J. A., Gallander, J. F., \& Peng, A. C. (1977). Effect of maturation on the lipid content of Concord grapes. American Journal of Enology and Viticulture, 28, 241-244.

Del Rio, J. L. P., \& Kennedy, J. A. (2006). Development of proanthocyanidins in Vitis vinifera L. cv. Pinot noir grapes and extraction into wine. American Journal of Enology and Viticulture, 57, 125-132.

Delfini, C., \& Cervetti, F. (1991). Metabolic and technological factors affecting acetic acid production by yeasts during alcoholic fermentation. Viticultural and Enological Sciences, 46, 142-150.

Della Corte, A., Chitarrini, G., Di Gangi, I. M., Masuero, D., Soini, E., Mattivi, F., \& Vrhovsek, U. (2015). A rapid LC-MS/MS method for quantitative profiling of fatty acids, sterols, glycerolipids, glycerophospholipids and sphingolipids in grapes. Talanta, 140, 52-61.

Erlund, I. (2004). Review of the flavonoids quercetin, hesperetin, and naringenin. Dietary sources, bioactivities, bioavailability, and epidemiology. Nutrition Research, 24, $851-874$.

Fahy, E., Cotter, D., Sud, M., \& Subramaniam, S. (2011). Lipid classification, structures and tools. Biochimica et Biophysica Acta, 1811, 637-647.

Gallander, J. F., \& Peng, A. C. (1980). Lipid and fatty acid compositions of different grape types. American Journal of Enology and Viticulture, 31, 24-27.

Gris, E. F., Mattivi, F., Ferreira, E. A., Vrhovsek, U., Pedrosa, R. C., \& Bordignon-Luiz, M. T. (2011). Proanthocyanidin profile and antioxidant capacity of Brazilian Vitis vinifera red wines. Food Chemistry, 126, 213-220.

Hilbert, G., Temsamani, H., Bordenave, L., Pedrot, E., Chaher, N., Cluzet, S., ... Richard, T. (2015). Flavonol profiles in berries of wild Vitis accessions using liquid chromatography coupled to mass spectrometry and nuclear magnetic resonance spectrometry. Food Chemistry, 169, 49-58.

Jeandet, P., Delaunois, B., Conreux, A., Donnez, D., Nuzzo, V., Cordelier, S., ... Courot, E. (2010). Biosynthesis, metabiolism, molecular engineering, and biological functions of stilbene phytoalexins in plants. BioFactors, 36, 331-341. 
Lafon-Lafourcade, S., Larue, F., \& Ribéreau-Gayon, P. (1979). Evidence for the existence of "survival factors" as an explanation for some peculiarities of yeast growth, especially in grape must of high sugar concentration. Applied and Environmental Microbiology, $38,1069-1073$

Lesschaeve, I., \& Noble, A. C. (2005). Polyphenols: Factors influencing their sensory properties and their effects on food and beverage preferences. The American Journal of Clinical Nutrition, 81, 330-335.

Liang, Z., Owens, C. L., Zhong, G. Y., \& Cheng, L. (2011). Polyphenolic profiles detected in the ripe berries of Vitis vinifera germplasm. Food Chemistry, 129, 940-950.

Liang, Z., Yang, Y., Cheng, L., \& Zhong, G. Y. (2012). Polyphenolic composition and content in the ripe berries of wild Vitis species. Food Chemistry, 132, 730-738.

Malacarne, G., Vrhovsek, U., Zulini, L., Cestaro, A., Stefanini, M., Mattivi, F., ... Moser, C. (2011). Resistance to Plasmopara viticola in a grapevine segregating population is associated with stilbenoid accumulation and with specific host transcriptional responses. BMC Plant Biology, 11, 1-13.

Mattivi, F., Guzzon, R., Vrhovsek, U., Stefanini, M., \& Velasco, R. (2006). Metabolite profiling of grape: Flavonols and anthocyanins. Journal of Agricultural and Food Chemistry, 54, 7692-7702.

Mattivi, F., Vrhovsek, U., Masuero, D., \& Trainotti, D. (2009). Differences in the amount and structure of extractable skin and seed tannins amongst red grape varieties. Australian Journal of Grape and Wine Research, 15, 27-35.

Mazzuca, P., Ferranti, P., Picariello, G., Chianese, L., \& Addeo, F. (2005). Mass spectrometry in the study of anthocyanins and their derivatives: Differentiation of Vitis vinifera and hybrid grapes by liquid chromatography/electrospray ionization mass spectrometry and tandem mass spectrometry. Journal of Mass Spectrometry, 40, 83-90.

Murtagh, F. \& Legendre, P. (2014). Ward's hierarchical agglomerative clustering method: Which algorithms implement Ward's criterion? Journal of Classification, 31, 274-295.

Narduzzi, L., Stanstrup, J., \& Mattivi, F. (2015). Comparing wild American grapes with Vitis vinifera: A metabolomics study of grape composition. Journal of Agricultural and Food Chemistry, 63, 6823-6834.

Pezet, R., Gindro, K., Viret, O., \& Spring, J. L. (2004a). Glycosylation and oxidative dimerization of resveratrol are respectively associated to sensitivity and resistance of grapevine cultivars to downy mildew. Physiological and Molecular Plant Pathology, 65, 297-303.

Pezet, R., Gindro, K., Viret, O., \& Richter, H. (2004b). Effects of resveratrol, viniferins and pterostilbene on Plasmopara viticola zoospore mobility and disease development. Vitis, 43, 145-148.

R Development Core Team (2014). R: A language and environment for statistical computing. Vienna, Austria: R Foundation for Statistical Computing.
Springer, L. F., Sherwood, R. W., \& Sacks, G. L. (2016). Pathogenesis-related proteins limit the retention of condensed tannin additions to red wines. Journal of Agricultural and Food Chemistry, 64, 1309-1317

Stacklies, W., Redestig, H., Scholz, M., Walther, D., \& Selbig, J. (2007). pcaMethods-A bioconductor package providing PCA methods for incomplete data. Bioinformatics, 23, 1164-1167.

Strimmer, K. (2008a). fdrtool: A versatile R package for estimating local and tail areabased false discovery rates. Bioinformatics, 24, 1461-1462.

Strimmer, K. (2008b). A unified approach to false discovery rate estimation. BMC Bioinformatics, 9, 303.

Sun, O., Gates, M. J., Lavin, E. H., Acree, T. E., \& Sacks, G. L. (2011). Comparison of odor-active compounds in grapes and wines from Vitis vinifera and non-foxy American grape species. Journal of Agricultural and Food Chemistry, 59, 10657-10664.

Teixeira, A., Eiras-Dias, J., Castellarin, S. D., \& Gerós, H. (2013). Berry phenolics of grapevine under challenging environments. International Journal of Molecular Sciences, 14, 18711-18739.

Tumanov, S., Zubenko, Y., Greven, M., Greenwood, D. R., Shmanai, V., \& Villas-Boas, S. G. (2015). Comprehensive lipidome profiling of Sauvignon blanc grape juice. Food Chemistry, 180, 249-256.

Vivier, M. a., \& Pretorius, I. S. (2000). Genetic improvement of grapevine: Tailoring grape varieties for the third millennium-A review. South African Journal of Enology and Viticulture, 21, 5-26.

Vrhovsek, U., Rigo, A., Tonon, D., \& Mattivi, F. (2004). Quantitation of polyphenols in different apple varieties. Journal of Agricultural and Food Chemistry, 52, 6532-6538.

Vrhovsek, U., Masuero, D., Gasperotti, M., Franceschi, P., Caputi, L., Viola, R., \& Mattivi, F. (2012). A versatile targeted metabolomics method for the rapid quantification of multiple classes of phenolics in fruits and beverages. Journal of Agricultural and Food Chemistry, 60, 8831-8840.

Wold $\mathrm{H}$. (1966). Estimation of principal components and related models by iterative least squares. In P. R. Krishnaiah (Ed.), Multivariate analysis (pp. 391-420). New York: Academic Press.

Xia, E. -Q., Deng, G. -F., Guo, Y. -J., \& Li, H. -B. (2010). Biological activities of polyphenols from grapes. International Journal of Molecular Sciences, 11, 622-646.

Zhao, Q., Duan, C. Q., \& Wang, J. (2010). Anthocyanins profile of grape berries of Vitis amurensis, its hybrids and their wines. International Journal of Molecular Sciences, 11, 2212-2218.

Zhu, L., Zhang, Y., \& Lu, J. (2012). Phenolic contents and composition in skins of red wine grape cultivars among various genetic backgrounds and originations. International Journal of Molecular Sciences, 13, 3492-3510. 